\title{
Increased expression of tumor protein D54 is associated with clinical progression and poor prognosis in patients with prostate cancer
}

\author{
LIGANG REN ${ }^{*}$, JING CHEN ${ }^{*}$ and XINNAN ZHANG \\ Department of Urology, Tongde Hospital of Zhejiang, Hangzhou, Zhejiang 310012, P.R. China
}

Received July 2, 2016; Accepted August 10, 2017

DOI: 10.3892/ol.2017.7214

\begin{abstract}
Tumor protein D54 (TPD54) has been reported to be involved in the prognosis of several cancers. However, the involvement of TPD54 in prostate cancer (PCa) is yet to be elucidated. In the present study, 117 patients with $\mathrm{PCa}$ were enrolled. The mRNA and protein levels of TPD54 in PCa tissues and adjacent normal prostate tissues were analyzed by quantitative polymerase chain reaction and western blotting. TPD54 expression was also determined by immunohistochemistry (IHC) in paraffin-embedded PCa tissues. The association between TPD54 expression and clinicopathological features and prognosis was evaluated. The results revealed that the expression levels of TPD54 mRNA and protein were upregulated in PCa tissues compared with adjacent normal prostate tissues. In addition, moderate/strong staining of TPD54 was observed in $91.4 \%(107 / 117)$ of PCa tissues, but only in $32.5 \%(38 / 117)$ of adjacent normal prostate tissues, as assessed by IHC. TPD54 expression was significantly associated with Gleason score $(\mathrm{P}=0.0001)$. In addition, patients with $\mathrm{PCa}$ with moderate/strong TPD54 expression had shorter biochemical recurrence-free survival times compared with those with negative/weak TPD54 expression $(\mathrm{P}=0.002)$. Multivariate analysis indicated that TPD54 overexpression was an independent prognostic factor for patients with PCa (hazard ratio, 2.259; 95\% confidence interval, 1.09-4.679; $\mathrm{P}=0.028$ ). Taken together, these results indicated that TPD54 is a predictor of poor outcome for patients with $\mathrm{PCa}$, and may be a potential prognostic marker for patients with $\mathrm{PCa}$.
\end{abstract}

\section{Introduction}

Prostate cancer (PCa) is a common malignancy of the urinary system in men aged $>50$ years old. In 2012, PCa was the

Correspondence to: Dr Ligang Ren, Department of Urology, Tongde Hospital of Zhejiang, 234 Gucui Road, Hangzhou, Zhejiang 310012, P.R. China

E-mail: ligangren77@sina.com

${ }^{*}$ Contributed equally

Key words: tumor protein D54, prostate cancer, prognosis second leading cause of cancer-associated mortality in the USA (1). The incidence of PCa has continued to increase in the past decades due to the wide use of serum prostate-specific antigen (PSA) screening throughout the world (2). Although the survival rates for patients with $\mathrm{PCa}$ are high, 30-40\% of patients experience PSA recurrence within 10 years of surgery or radiation treatment (3). However, previous studies have raised concern that PSA may not be an optimal prognostic biomarker. For example, the overall sensitivity of PSA may be too low to predict the morbidity and mortality rate accurately (4). By contrast, since the sensitivity of PSA is too low, false-positive diagnosis of PCa commonly occurs and results in over-detecting and overtreating, with, the majority of males with PCa succumbing to unrelated causes of mortality (5). Therefore, there is an urgent need to identify novel, robust biomarkers for PCa.

Tumor protein D54 (TPD54), also termed TPD52L2, is the third identified member of the tumor protein D52 (TPD52) family (6). The family members are characterized by an $\mathrm{N}$-terminal coiled-coil motif that is used to form homo- and heterologous complexes with other tumor protein D52-1ike proteins (7). The most important physiological function of this family protein is its involvement in $\mathrm{Ca}^{+}$-dependent vesicular transport processes (8). A previous study demonstrated that TPD52 is highly expressed in multiple types of human solid tumor, including lung, colon, ovary and breast cancer (9), which indicates that TPD52 family members may serve as novel markers of malignant tumors. A previous study demonstrated that TPD54 is a negative regulator of extracellular matrix-dependent migration and attachment in oral squamous cell carcinoma (10). In addition, lentivirus-mediated knockdown of TPD54 inhibits cell proliferation of glioma (11), oral squamous cell carcinoma (OSCC) (12), gastric cancer (13), breast cancer (14) and liver cancer (15) cells in vitro. The association between the expression of TPD54 and clinical outcome in prostate cancer (16) and breast childhood leukemia (17) has also been reported. In spite of these results, however, the associations between TPD54 and the clinicopathological characteristics of prostate cancer have not been fully explored.

The present study aimed to evaluate the expression of TPD54 in PCa tumor tissues and adjacent noncancerous tissues, and to compare TPD54 expression with clinicopathological characteristics. Finally, the value of TPD54 as a prognostic biomarker for patients with PCa was evaluated. 


\section{Materials and methods}

Patients and tissue samples. The present study was conducted in accordance with the ethical principles for human experimentation stated in the Declaration of Helsinki and all applicable amendments that have been defined by the International Conference of Harmonization Good Clinical Practice Guidelines (E6 R2), ensuring that the rights, safety and well-being of the subjects are safeguarded and that the integrity of the data acquired during the study was preserved. The study protocol was approved by the Ethics Committee of the Tongde Hospital of Zhejiang Province (Zhejiang, China; approval number: 2012-025) and written informed consent was obtained for all patients prior to their participation in the present study. The present study consisted of samples from 117 patients with $\mathrm{PCa}$ who underwent radical retropubic prostatectomy between January 2011 and January 2013 at the Department of Urology, Tongde Hospital of Zhejiang Province. All the samples were obtained with informed consent from the patients, which was provided prior to surgery. The clinicopathological characteristics of patients, including age at diagnosis, preoperative PSA, postoperative pathological state, Gleason score, pathological T stage, capsule penetration, surgical margins, perineural invasion and seminal vesicle invasion are presented in Table I. All the diagnoses of patients were confirmed by pathologists. All of the tumor specimens consisted of primary prostate tumor tissues and adjacent normal prostate tissues, and each specimen was divided into two parts in a standard process. Briefly, surgically resected specimens were placed in the correct orientation. The prostate was cut along the coronal plane into 6-8 parts; each section was $\sim 5 \mathrm{~mm}$ thick. PCa tissues and adjacent normal tissues were carefully recognized and cut into two parts, and each specimen was divided into two parts along the coronal plane. One was snap-frozen in liquid nitrogen, and stored at $-180^{\circ} \mathrm{C}$ for extraction of RNA, while the other part of the specimen was fixed in $10 \%$ buffered formalin at room temperature overnight for immunohistochemistry (IHC). A total of 111 patients with PCa were successfully followed-up, whereas 6 patients who succumbed to diseases other than PCa or from unexpected events were excluded. The mean follow-up time was 42 (9-61) months. For the analysis of biochemical recurrence (BCR)-free survival, the date of prostatectomy was used to represent the beginning of the follow-up period. The endpoint was the time to biochemical relapse, which was defined as the period between surgical treatment and two consecutive measurements of serum PSA $\geq 0.2 \mathrm{ng} / \mathrm{ml}$.

Reverse transcription-quantitative polymerase chain reaction $(R T-q P C R)$. Total RNA from the primary tumor and adjacent normal tissues was extracted using TRIzol reagent (Thermo Fisher Scientific, Inc., Waltham, MA, USA) according to the manufacturer's protocol. RNA $(1 \mu \mathrm{g})$ from each sample was used for cDNA synthesis using M-MLV Reverse Transcriptase (Thermo Fisher Scientific, Inc.) according to the manufacturer's protocol. The reverse transcription system was as follows: Oligo $(\mathrm{dT})_{12-18}(1 \mu \mathrm{l})$, total RNA $(1 \mu \mathrm{g}), 10 \mathrm{mM} \mathrm{dNTP}(1 \mu \mathrm{l})$ and distilled water up to $12 \mu \mathrm{l}$. The mixture was heated to $65^{\circ} \mathrm{C}$ for $5 \mathrm{~min}$ and chilled on ice. To the mixture was then added 5X First-Strand Buffer (4 $\mu \mathrm{l}), 0.1$ M DTT $(2 \mu \mathrm{l})$, recombinant ribonuclease inhibitor ( $1 \mu 1)$, M-MLV RT (1 $\mu 1)$, which was mixed and incubated for $50 \mathrm{~min}$ at $37^{\circ} \mathrm{C}$. The mRNA levels were quantified by qPCR with the Applied Biosystems 7500 PCR system using SYBR Master Mix (Thermo Fisher Scientific, Inc.). The sequences of the primers were as follows: TPD54 forward, 5'-CATGACGTGCAGGTCTCTAGC-3' and reverse, 5'-GCCTGTGAAAGAGTTTCCTGAGT-3'; GAPDH forward, 5'-GCACCGTCAAGGCTGAGAAC-3' and reverse, 5'-TGGTGAAGACGCCAGTGGA-3'. The PCR conditions were as follows: $50^{\circ} \mathrm{C}$ for $2 \mathrm{~min}$ and $95^{\circ} \mathrm{C}$ for $10 \mathrm{~min}$, followed by 40 cycles at $95^{\circ} \mathrm{C}$ for $15 \mathrm{sec}$ and $60^{\circ} \mathrm{C}$ for 1 min. Relative quantification of TPD54 mRNA expression was calculated using the $2^{-\Delta \Delta \mathrm{Cq}}$ method (17). Normalization was performed against GAPDH mRNA expression.

Western blotting. Total proteins from 8 pairs of fresh prostate cancer and adjacent normal tissues were extracted using RIPA buffer (cat. no., 9806; Cell Signaling Technology, Inc., Danvers, MA, USA). Briefly, tissues were cut into small pieces, and $20 \mathrm{mg}$ of tissue was homogenized with a glass tissue grinder in 100-200 $\mu$ l RIPA buffer on ice for $30 \mathrm{~min}$. Lysates were then centrifuged at $8,000 \mathrm{x}$ g at $4^{\circ} \mathrm{C}$ for $20 \mathrm{~min}$, and the supernatants were collected. The protein concentrations were determined using a bicinchoninic acid assay kit (Beyotime Institute of Biotechnology, Haimen, China). Next, equal amounts of loading buffer were added and the protein was boiled. Proteins were resolved using a $12 \%$ gel and SDS-PAGE, transferred to polyvinylidene fluoride membranes and incubated with the following antibodies: Anti-TPD52L2 (dilution, 1:500; cat. no., 11795-1-AP, ProteinTech Group, Inc., Chicago, IL, USA) and anti- $\beta$-actin (dilution, 1:2,000; cat. no., 60008-1-Ig, ProteinTech Group, Inc.) at room temperature for $2 \mathrm{~h}$. Following washing with TBS-Tween-20 (TBST) three times, the membranes were incubated with corresponding horseradish peroxidase-conjugated goat anti-mouse $\operatorname{IgG}$ (dilution, 1:2,000; cat. no. A0216; Beyotime Institute of Biotechnology) or goat anti-rabbit IgG (dilution, 1:2,000; cat. no. A0239; Beyotime Institute of Biotechnology) antibodies were used as secondary antibodies for $1 \mathrm{~h}$ at room temperature, and then washed three times with TBST. The final band was visualized using enhanced chemiluminescence reagents (Thermo Fisher Scientific, Inc.) and detected by an Alpha Imager v.FC800 (Alpha Innotech, San Leandro, CA, USA). The statistical analyses were conducted from three independent experiments.

Immunohistochemical staining. Paraffin-embedded samples were cut into $4-\mu \mathrm{m}$ thick sections and stained with hematoxylin and eosin for $10 \mathrm{~min}$ at room temperature for tumor confirmation. The expression level of TPD52L2 was determined using a Strept Avidin-Biotin Complex immunohistochemical assay kit (catalog no. SA1027, Wuhan Boster Biological Technology, Ltd., Wuhan, China) according to the manufacturer-s protocol. Briefly, antigen retrieval was performed by immersing the slides in a solution of $0.01 \%$ sodium citrate $\mathrm{pH} 6.0$ for $5 \mathrm{~min}$ in boiling water. Endogenous peroxidase activity was inhibited by immersing the slides in $3 \% \mathrm{H}_{2} \mathrm{O}_{2}$-methanol at room temperature for $10 \mathrm{~min}$ and then incubated in 5\% goat antiserum (cat. no. C0265; Beyotime Institute of Biotechnology) for $15 \mathrm{~min}$ at $37^{\circ} \mathrm{C}$. The sections were then sequentially incubated overnight at $4^{\circ} \mathrm{C}$ with the rabbit anti-human TPD52L2 polyclonal 
Table I. Clinicopathological characteristics of 117 patients with prostate cancer.

\begin{tabular}{lc}
\hline Features & Patients \\
\hline Median age (range), years & $68(42-80)$ \\
PSA, ng/ml & \\
$<10$ & $34(29)$ \\
$10-20$ & $33(28)$ \\
$>20$ & $50(43)$ \\
GS & \\
$<7$ & $41(35)$ \\
$=7$ & $45(38)$ \\
$>7$ & $31(26)$ \\
pT stage & \\
pT2 & $97(83)$ \\
pT3 & $20(17)$ \\
pN stage & \\
N0 & $109(93)$ \\
N1 & $8(7)$ \\
CP & \\
+ & \\
- & $7(6)$ \\
SM & $110(94)$ \\
+ & \\
- & $103(88)$ \\
PNI & \\
+ & \\
SVI & \\
+ & \\
\hline
\end{tabular}

PSA, prostate specific antigen; GS, Gleason score; pT stage, pathological $\mathrm{T}$ stage; $\mathrm{pN}$ stage, pathological $\mathrm{N}$ stage; $\mathrm{CP}$, capsule penetration; SM, surgical margins; PNI, perineural invasion; SVI, seminal vesicle invasion.

antibody (dilution, 1:100; cat. no., 11795-1-AP, ProteinTech Group, Inc.), biotinylated goat anti-rabbit immunoglobulin $\mathrm{G}$ (cat. no. TA130016; dilution, 1:1,000; OriGene Technologies, Inc., Beijing, China) and avidin biotin-peroxidase complex. Following staining with diaminobenzidine for 3-10 min at room temperature, sections were analyzed under a light microscope (magnification, x100; Olympus BX-40; Olympus Corporation, Tokyo, Japan).

Immunohistochemical staining was evaluated independently by two pathologists in Department of Pathology, Tongde Hospital of Zhejiang Province (Hangzhou, China). The level of TPD54 staining was based on the intensity of staining and the proportion of positively stained cancer cells. Intensity of staining was scored as follows: 0 , no staining; 1 , light yellow; 2 , yellow brown; 3 , strong brown color. The proportion positive tumor cells was scored as follows: $0, \leq 5 \%$ positive tumor cells; $1,6<25 \%$ positive tumor cells; $2,26-50 \%$ positive tumor cells;

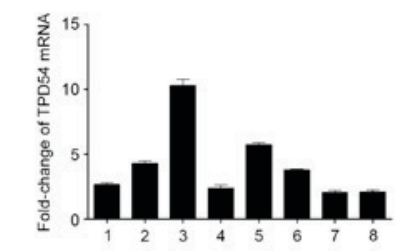

c

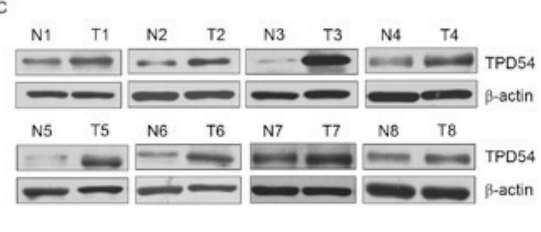

B

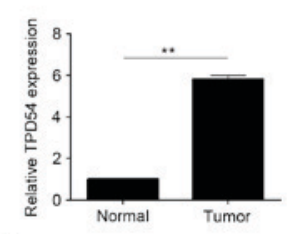

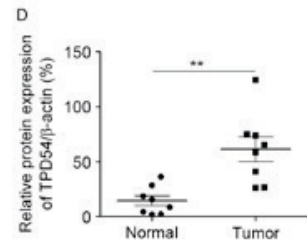

Figure 1. mRNA and protein expression of TPD54 in 8 randomly-chosen pairs of prostate cancer tissues and adjacent normal prostate tissues. (A) Fold change of TPD54 mRNA in the 8 tissues, and (B) relative TPD54 mRNA expression. (C) Western blot analysis of TPD54 protein expression, with (D) quantification. ${ }^{* *} \mathrm{P}<0.001$ vs. the normal tissues. TPD45, tumor protein D45.

$3,51-75 \%$ positive tumor cells and $4, \geq 76 \%$ positive tumor cells. The final basis (IS; immunoreactivity score) for grouping was the product of the staining area score and staining intensity as follows: Negative (-), score 0; weak (+), scores 1-4; moderate $(++)$, scores 5-8; strong positive $(++)$, scores 9-12.

Statistical analysis. Statistical analyses were performed using SPSS 19.0 software (IBM SPSS, Armonk, NY, USA). Results are expressed as the mean \pm standard deviation. The differences in TPD54 expression between PCa and adjacent normal prostate tissues were analyzed by the non-parametric Mann-Whitney U-test. The distribution of TPD54 expression in patients with PCa to clinicopathological features was analyzed using non-parametric Kruskal-Wallis one-way ANOVA and Dunn's post hoc test. Univariate and multivariate Cox regression analyses were performed to analyze the survival data. Survival curves were plotted using the Kaplan-Meier method, and differences were tested using the log-rank test. $\mathrm{P}<0.05$ was considered to indicate a statistically significant difference.

\section{Results}

TPD54 protein is overexpressed in patients with PCa. The mRNA and protein expression of TPD54 was initially examined by RT-qPCR and western blotting in 8 randomly selected pairs of primary prostate cancer and adjacent normal prostate tissues. As presented in Fig. 1, a significant increase in mRNA and protein expression of TPD54 was observed in prostate cancer tissues compared with adjacent normal tissues. In addition, TPD54 expression was evaluated in 117 paired prostate cancer cases by immunohistochemistry (IHC). The IHC data revealed that TPD54 was mainly localized in the cytoplasm of the prostate cancer cells, and immunoreactivity ranged between 0 and $100 \%$ (Fig. 2). According to the IHC score analysis, in prostate tumor tissues, $1 / 117(0.9 \%)$ exhibited negative TPD54 staining, 9/117 (7.7\%) weak staining, 75/117 (64.1\%) moderate staining and 32/117 (27.3\%) strong staining; whereas in adjacent normal prostate tissues, 15/117 (12.8\%) demonstrated negative TPD54 staining, 64/117 (51.7\%) weak staining, 37/117 (31.6\%) moderate staining and 1/117 (0.9\%) strong staining. The Mann-Whitney $U$ test revealed that the 
Table II. Association of intra-tumoral tumor protein D54 expression and clinicopathological variables of 117 patients with prostate cancer.

\begin{tabular}{|c|c|c|c|c|c|}
\hline \multirow[b]{2}{*}{ Variables } & \multicolumn{4}{|c|}{ TPD54 expression, $\mathrm{n}$} & \multirow[b]{2}{*}{ P-value } \\
\hline & Negative & Weak & Moderate & Strong & \\
\hline \multicolumn{6}{|c|}{ Age, years } \\
\hline$<65$ & 0 & 3 & 23 & 12 & 0.738 \\
\hline $65-75$ & 1 & 4 & 36 & 13 & \\
\hline$>75$ & 0 & 2 & 13 & 7 & \\
\hline \multicolumn{6}{|c|}{$\mathrm{PSA}, \mathrm{ng} / \mathrm{ml}$} \\
\hline$<10$ & 0 & 5 & 21 & 8 & 0.126 \\
\hline $10-20$ & 0 & 4 & 22 & 7 & \\
\hline$>20$ & 1 & 0 & 32 & 17 & \\
\hline \multicolumn{6}{|l|}{ GS } \\
\hline$<7$ & 1 & 7 & 25 & 8 & 0.005 \\
\hline$=7$ & 0 & 2 & 33 & 10 & \\
\hline$>7$ & 0 & 0 & 17 & 14 & \\
\hline \multicolumn{6}{|l|}{ pT stage } \\
\hline pT2 & 1 & 5 & 63 & 28 & 0.136 \\
\hline pT3 & 0 & 4 & 12 & 4 & \\
\hline \multicolumn{6}{|l|}{ pN stage } \\
\hline N0 & 1 & 9 & 69 & 30 & 0.148 \\
\hline $\mathrm{N} 1$ & 0 & 0 & 6 & 2 & \\
\hline \multicolumn{6}{|l|}{$\mathrm{CP}$} \\
\hline+ & 0 & 1 & 5 & 1 & 0.381 \\
\hline- & 1 & 8 & 70 & 31 & \\
\hline \multicolumn{6}{|l|}{ SM } \\
\hline+ & 0 & 2 & 7 & 5 & 0.770 \\
\hline- & 1 & 7 & 68 & 27 & \\
\hline \multicolumn{6}{|l|}{ PNI } \\
\hline $\begin{array}{l}+ \\
-\end{array}$ & 0 & 0 & 16 & 8 & 0.191 \\
\hline 1 & & 9 & 59 & 24 & \\
\hline \multicolumn{6}{|l|}{ SVI } \\
\hline+ & 0 & 1 & 13 & 4 & 0.816 \\
\hline- & 1 & 8 & 62 & 28 & \\
\hline
\end{tabular}

PSA, prostate specific antigen; GS, Gleason score; pT stage, pathological $\mathrm{T}$ stage; $\mathrm{pN}$ stage, pathological $\mathrm{N}$ stage; $\mathrm{CP}$, capsule penetration; SM, surgical margins; PNI, perineural invasion; SVI, seminal vesicle invasion.

expression level of TPD54 in prostate cancer tissues was significantly higher than that in adjacent normal prostate tissues $(\mathrm{P}=0.0001)$. Taken together, these results demonstrated that TPD54 expression was upregulated in PCa tissues, and may be involved in PCa progression.

TPD54 expression is associated with clinical data in patients with PCa. Whether TPD54 expression was associated with patient-associated clinical factors was subsequently explored.
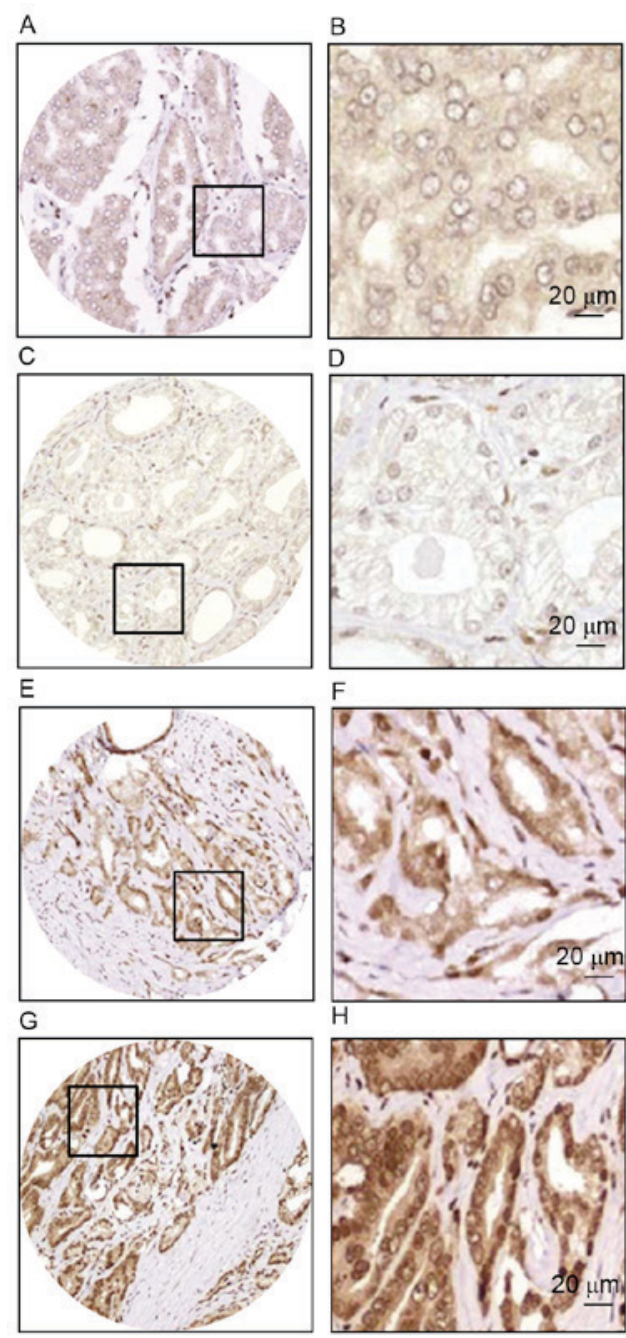

Figure 2. Immunohistochemical staining of TPD54 in prostate cancer tissues. Negative staining at (A) x100 and (B) x400 magnification, weak staining at (C) x100 and (D) x400 magnification, moderate staining at (E) x100 and (F) x400 magnification, and strong staining at (G) x100 and (H) x400 magnification (scale bar, $20 \mu \mathrm{m}$ ). TPD45, tumor protein D45.

The associations between TPD54 expression and clinicopathological features are summarized in Table II. The results indicated that TPD54 expression was significantly associated with Gleason score $(\mathrm{P}=0.0001)$. However, no significant associations were identified with age, preoperative PSA, pathological $\mathrm{T}$ stage, postoperative pathological state, capsule penetration, surgical margins, perineural invasion and seminal vesicle invasion. These data demonstrated that increased TPD54 expression may promote tumor growth of PCa.

Associations between TPD54 expression and prognosis in patients with $\mathrm{PCa}$. To determine whether TPD54 expression is a prognostic factor for patients with $\mathrm{PCa}$, Kaplan-Meier analysis and log-rank tests were performed to analyze TPD54 expression and clinical follow-up data in 111 patients. Of the 111 patients with $\mathrm{PCa}$, biochemical recurrence occurred in 27 cases (24.3\%). The results demonstrated that the BCR-free survival rate for patients with strong TPD54 expression was 56.3\%, which was significantly lower than that in patients with moderate TPD54 expression $(82.7 \% ; \mathrm{P}=0.009)$ and patients with weak/negative TPD54 expression (100.0\%). These results revealed that a high 
Table III. Univariate and multivariate analyses of factors associated with biochemical recurrence-free survival of patients with prostate cancer.

\begin{tabular}{|c|c|c|c|c|c|c|}
\hline \multirow[b]{2}{*}{ Factors } & \multicolumn{3}{|c|}{ Univariate analysis } & \multicolumn{3}{|c|}{ Multivariate analysis } \\
\hline & Hazard ratio & $95 \%$ CI & P-value & Hazard ratio & $95 \% \mathrm{CI}$ & P-value \\
\hline Age, years $(\leq 70,>70)$ & 1.941 & $0.908-4.149$ & 0.087 & & & \\
\hline PSA, ng/ml $(<10,10-20,>20)$ & 2.166 & $1.268-3.698$ & 0.005 & 2.047 & $1.547-4.269$ & 0.037 \\
\hline $\mathrm{GS}(<7,7,>7)$ & 4.555 & $2.423-8.566$ & $<0.001$ & 3.832 & $1.966-7.469$ & $<0.001$ \\
\hline pT Stage (pT2, pT3) & 5.649 & $2.631-12.129$ & $<0.001$ & 8.097 & $3.606-18.180$ & $<0.001$ \\
\hline pN Stage (pN0, pN1) & 7.858 & $3.090-19.983$ & $<0.001$ & 7.694 & $3.897-10.670$ & $<0.001$ \\
\hline $\mathrm{CP}(+,-)$ & 1.328 & $0.314-5.619$ & 0.700 & & & \\
\hline $\mathrm{SM}(+,-)$ & 1.885 & $0.713-4.985$ & 0.201 & & & \\
\hline PNI $(+,-)$ & 2.842 & $1.291-6.258$ & 0.009 & 2.364 & $1.841-5.632$ & 0.013 \\
\hline $\operatorname{SVI}(+,-)$ & 1.239 & $0.469-3.276$ & 0.666 & & & \\
\hline TPD54 expression $(-,+,++,++)$ & 2.790 & $1.407-5.534$ & 0.003 & 2.259 & $1.090-4.679$ & 0.028 \\
\hline
\end{tabular}

PSA, prostate specific antigen; GS, Gleason score; pT stage, pathological T stage; pN stage, pathological N stage; CP, capsule penetration; SM, surgical margins; PNI, perineural invasion; SVI, seminal vesicle invasion.

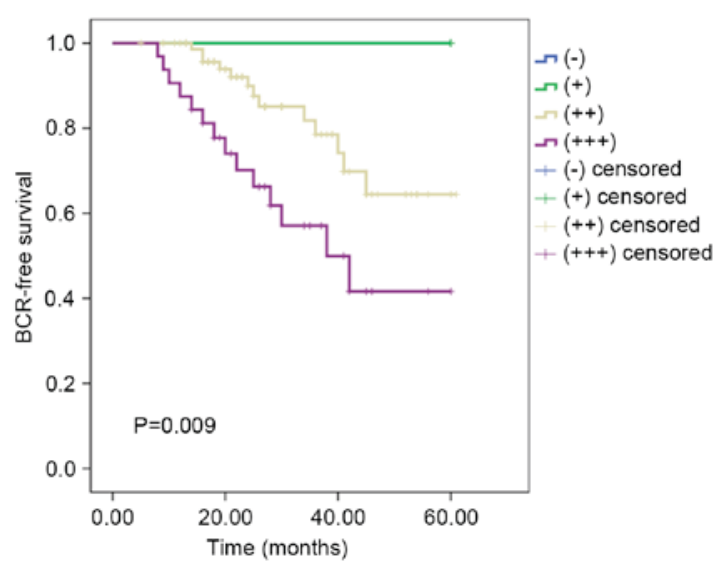

Figure 3. BCR-free survival curve based on tumor protein D54 expression in patients with prostate cancer. P-values were determined by the log-rank test. $\mathrm{BCR}$, biochemical recurrence.

level of TPD54 expression is a negative indicator of prognosis for patients with PCa (Fig. 3). In addition, univariate and multivariate analyses revealed that TPD54 expression, PSA, Gleason score, $\mathrm{pT}$ stage, $\mathrm{pN}$ stage, perineural invasion were all independent prognostic factors in patients with PCa (Table III). Therefore, the present results demonstrated that TPD54 may be a significant prognostic marker for patients with $\mathrm{PCa}$.

\section{Discussion}

At present, radical prostatectomy is one of the most effective methods for the treatment of localized PCa (2). However, a significant percentage of prostate tumors progress rapidly and this is responsible for the poor survival of patients, irrespective of active treatment (3). Therefore, identification of PCa-specific biomarkers may be important for diagnosis, therapy and prognostic prediction. To the best of our knowledge, the present study has demonstrated, for the first time, that the expression of TPD54 in 117 patients with PCa is associated with patient survival and clinicopathological characteristics. Positive TPD54 expression was observed in $91.4 \%$ of PCa tissues in the present study. Furthermore, a significant association was observed between positive TPD54 expression and poor prognosis, independent of other patient characteristics. These results indicated that TPD54 expression may be a novel prognostic marker for PCa.

TPD54, which was upregulated in several types of solid malignancies, is an oncogene responsible for the high proliferation rates observed in cancer cells (9). Several studies have reported that the suppression of TPD54 gene inhibits the growth of tumor cells. TPD54 was overexpressed in OSCC-derived cell lines. Knockdown of TPD54 in OSCC cell lines induces cell growth inhibition, promotes cell apoptosis, and inhibits extracellular matrix-dependent cell migration and attachment in OSCC $(10,12)$. Furthermore, Yang et al (14) reported that Lentivirus-mediated TPD54 inhibited proliferation and colony formation in breast cancer cell line, ZR-75-30. Furthermore, knockdown of TPD54 promoted glycogen synthase kinase-3 $\beta$ phosphorylation in ZR-75-30 cells. Similar results were observed in glioma (11), gastric cancer (13) and liver cancer (15). Previous studies have also reported the association between the expression of TPD54 and clinical outcome in PCa (16) and childhood leukemia (17). Accordingly, in the present study, TPD54 mRNA expression in PCa samples was investigated using qPCR analysis and TPD54 protein expression was investigated using western blot analysis. The results demonstrated that TPD54 mRNA and protein levels were significantly increased in tumor tissues compared with those in the adjacent normal prostate tissues. These results indicated that TPD54 may serve a critical function in PCa progression. However, to learn more about the biological function of TPD54 in PCa development, knockdown or overexpression assays in PCa cell lines are urgently required.

In the present study, TPD54 protein expression was analyzed in tumor tissues by IHC in order to assess its prognostic significance for PCa. IHC analysis revealed moderate/strong TPD54 
expression in $91.4 \%(107 / 117)$ of patients with PCa, markedly higher than that in adjacent normal prostate tissues $(32.5 \%$, 38/117). High expression of TPD54 was revealed to be significantly associated with the Gleason score of patients with $\mathrm{PCa}$, indicating that TPD54 expression may promote tumor growth of PCa. These results suggested that TPD54 may be involved in the tumorigenesis and progression of PCa. Furthermore, the results demonstrated that patients with high TPD54 expression presented with a shorter BCR-free survival time compared with those with low TPD54 expression. Univariate analyses revealed that increased TPD54 expression in PCa tissues was significantly associated with BCR-free survival. In addition, multivariate analysis revealed that TPD54 expression was an independent prognostic factor for patients with $\mathrm{PCa}$. These results suggested that TPD54 may be an important prognostic marker for patients with PCa. Notably, using the Gene Expression Omnibus database and Top-scoring pairs algorithm, Zhao et al (16) revealed that TPD52L2/SQLE gene pair was a top-scoring pair for predicting prostate tumor progression.

In conclusion, the present study demonstrated that TPD54 was overexpressed in patients with $\mathrm{PCa}$, and its expression was associated with a worse clinical outcome. TPD54 may serve as a potential prognostic indicator for $\mathrm{PCa}$, but may not serve as an optimal biomarker for clinical use currently. Additional studies are required using a larger cohort of tumor samples to confirm the prognostic function of TPD54 in PCa, and translational and prospective studies of TPD54 as a therapeutic target in PCa are also required.

\section{References}

1. Torre LA, Bray F, Siegel RL, Ferlay J, Lortet-Tieulent J and Jemal A: Global cancer statistics, 2012. CA Cancer J Clin 65: 87-108, 2015.

2. Parnes HL, House MG and Tangrea JA: Prostate cancer prevention: Strategies for agent development. Curr Opin Oncol 25: 242-251, 2013.

3. Walz J, Joniau S, Chun FK, Isbarn H, Jeldres C, Yossepowitch O, Chao-Yu H, Klein EA, Scardino PT, Reuther A, et al: Pathological results and rates of treatment failure in high-risk prostate cancer patients after radical prostatectomy. BJU Int 107: 765-770, 2011.
4. Andriole GL, Crawford ED, Grubb RL III, Buys SS, Chia D, Church TR, Fouad MN, Gelmann EP, Kvale PA, Reding DJ, et al: Mortality results from a randomized prostate-cancer screening trial. N Engl J Med 360: 1310-1319, 2009.

5. Shariat SF, Semjonow A, Lilja H, Savage C, Vickers AJ and Bjartell A: Tumor markers in prostate cancer I: Blood-based markers. Acta Oncol 50 (Suppl 1): S61-S75, 2011.

6. Byrne JA, Nourse CR, Basset P and Gunning P: Identification of homo- and heteromeric interactions between members of the breast carcinoma-associated D52 protein family using the yeast two-hybrid system. Oncogene 16: 873-881, 1998.

7. Boutros R, Fanayan S, Shehata M and Byrne JA: The tumor protein D52 family: Many pieces, many puzzles. Biochem Biophys Res Commun 325: 1115-1121, 2004.

8. Thomas DD, Frey CL, Messenger SW, August BK and Groblewski GE: A role for tumor protein TPD52 phosphorylation in endo-membrane trafficking during cytokinesis. Biochem Biophys Res Commun 402: 583-587, 2010.

9. Tennstedt P, Bölch C, Strobel G, Minner S, Burkhardt L, Grob T, Masser S, Sauter G, Schlomm T and Simon R: Patterns of TPD52 overexpression in multiple human solid tumor types analyzed by quantitative PCR. Int J Oncol 44: 609-615, 2014.

10. Mukudai Y, Kondo S, Fujita A, Yoshihama Y, Shirota T and Shintani S: Tumor protein D54 is a negative regulator of extracellular matrix-dependent migration and attachment in oral squamous cell carcinoma-derived cell lines. Cell Oncol (Dordr) 36: 233-245, 2013.

11. Wang Z, Sun J, Zhao Y, Guo W, Lv K and Zhang Q: Lentivirus-mediated knockdown of tumor protein D52-like 2 inhibits glioma cell proliferation. Cell Mol Biol (Noisy-le-grand) 60: 39-44, 2014.

12. He Y, Chen F, Cai Y and Chen S: Knockdown of tumor protein D52-like 2 induces cell growth inhibition and apoptosis in oral squamous cell carcinoma. Cell Biol Int 39: 264-271, 2015.

13. Xu J, Wang W, Zhu Z, Wei Z, Yang D and Cai Q: Tumor protein D52-like 2 accelerates gastric cancer cell proliferation in vitro. Cancer Biother Radiopharm 30: 111-116, 2015.

14. Yang M, Wang X, Jia J, Gao H, Chen P, Sha X and Wu S: Tumor protein D52-like 2 contributes to proliferation of breast cancer cells. Cancer Biother Radiopharm 30: 1-7, 2015.

15. Pan ZY, Yang Y, Pan H, Zhang J, Liu H, Yang Y, Huang G, Yin L, Huang $J$ and Zhou WP: Lentivirus-mediated TPD52L2 depletion inhibits the proliferation of liver cancer cells in vitro. Int J Clin Exp Med 8: 2334-2341, 2015.

16. Zhao H, Logothetis CJ and Gorlov IP: Usefulness of the top-scoring pairs of genes for prediction of prostate cancer progression. Prostate Cancer Prostatic Dis 13: 252-259, 2010.

17. Barbaric D, Byth K, Dalla-Pozza L and Byrne JA: Expression of tumor protein D52-like genes in childhood leukemia at diagnosis: Clinical and sample considerations. Leuk Res 30: 1355-1363, 2006. 